\title{
Effect of Relaxin Expressing Adenovirus on Scar Remodeling: A Preliminary Study
}

Bok Ki Jung ${ }^{1}$, Won Jai Lee ${ }^{2}$, Eunhye Kang ${ }^{2}$, Hyo Min Ahn ${ }^{3}$, Yong Oock Kim², Dong Kyun Rah², Chae-Ok Yun ${ }^{3}$, In Sik Yun ${ }^{1}$

${ }^{1}$ Department of Plastic and Reconstructive Surgery, Gangnam Severance Hospital, Yonsei University College of Medicine, Seoul; ${ }^{2}$ Institute for Human Tissue Restoration, Department of Plastic and Reconstructive Surgery, Yonsei University College of Medicine, Seoul;

${ }^{3}$ Department of Bioengineering, College of Engineering, Hanyang University, Seoul, Korea
Background: Relaxin is a transforming growth factor $\beta 1$ antagonist. To determine the effects of relaxin on scar reduction, we investigated the scar remodeling process by injecting relaxin-expressing adenoviruses using a pig scar model.

Methods: Scars with full thickness were generated on the backs of Yorkshire pigs. Scars were divided into two groups (relaxin $[R L X]$ and Control). Adenoviruses were injected into the RLX (expressing relaxin) and Control (not expressing relaxin) groups. Changes in the surface areas, color index and pliability of scars were compared.

Results: Fifty days after treatment, the surface areas of scars decreased, the color of scars was normalized, and the pliability of scars increased in RLX group.

Conclusion: Relaxin-expressing adenoviruses improved the surface area, color, and pliability of scars. The mechanism of therapeutic effects on scar formation should be further investigated.

Keywords: Gene therapy / Cicatrix / Relaxin / Adenovirus
No potential conflict of interest relevant to this article was reported.

\section{INTRODUCTION}

Scars occurring as the result of wound healing are able to elicit functional disability due to secondary scar contracture in addition to aesthetic and structural disorders. In cases of burn scars, scar contracture, in particular, can generate severe functional problems in the facial or joint region [1]. Moreover, pathologic scars, such as keloids and hypertrophic scars, enlarge drastically,

\footnotetext{
Correspondence: In Sik Yun

Department of Plastic and Reconstructive Surgery, Gangnam Severance Hospital, Yonse University College of Medicine, 211 Eonju-ro, Gangnam-gu, Seoul 06273, Korea E-mail: eqatom@yuhs.ac

*This work was supported by a faculty research grant of Yonsei University College of Medicine (6-2012-0037, Dr, IS. Yun), grants from the Ministry of Knowledge Economy (10030051, Dr. C-O. Yun), the National Research Foundation of Korea (2010-0029220, 2013K1A1A2A02050188, 2013M3A9D3045879, Dr. C-O. Yun and NRF-2015R1D1A1A01060361, Dr. IS. Yun), and Korea Food and Drug Administration (KFDA-13172-356, Dr. C-O. Yun).
}

Received January 19, 2017 / Revised February 24, 2017 / February 24, 2017 resulting in an intractable disease, as it may relapse postoperatively. Fundamental treatments for scars have not yet been developed. In addition to wound healing, scar development remains an important research area in medicine. Although a large number of studies have investigated the mechanism of scar development to both minimize and alter scar formation, treatments for scars have not yet been fully realized [2].

Relaxin is a 6-kDa polypeptide hormone belonging to the insulin-like growth factor (IGF) family; it is primarily produced in the corpus luteum and endometrium [3]. Recent studies revealed that relaxin exhibits biological activation in reproductive tissues and other organs [4-6]. Relaxin has anti-fibrotic effects that reduce inflammation and collagen synthesis. Relaxin is also known to reduce the activation of transforming growth factor $\beta 1$ (TGF- $\beta 1$ ), which plays an important role in scar formation. Through this process, the differentiation of fibroblasts into myofibroblasts is 
anticipated to slow. This anti-fibrotic effect of relaxin is thought to reduce scar formation. In addition, the effects of relaxin on mast cells, which play crucial roles in scar formation, must be verified [7-9].

To overcome the very short biological half-life of relaxin $(<4$ minutes), adenoviruses expressing the relaxin gene were used as vectors. Gene therapy using adenoviruses has been primarily investigated for anti-tumor effects [10]. Instead of using the E1 gene, which is essential for adenovirus duplication, the relaxin gene was inserted into a non-duplicable adenovirus to prolong its effects by amplifying its expression both in and around adenovirus-infected cells. Since non-duplicable adenoviruses show anti-tumor effects in localized areas, they are considered to be suitable for the treatment of scars and other fibrotic diseases [10,11].

We aim to verify the effects of relaxin on scar remodeling by injecting relaxin-expressing adenoviruses in a pig scar model.

\section{METHODS}

\section{Scar formation}

The scar model was created using three Yorkshire pigs (4 months of age, $40 \mathrm{~kg}$ ). Anesthesia was induced in each pig via an intramuscular injection of Zoletil ( $5 \mathrm{mg} / \mathrm{kg}$, Virbac, Carros, France) and Rompun (2 mg/kg, Bayer, Seoul, Korea); all hairs were then removed from both the back and belly of each pig. Inhalation anesthesia was induced using isoflurane (IsoFlo, Abbott Laboratories, Abbott Park, IL, USA). After anesthetizing the pigs, a total of 36 full-thickness skin defects $\left(3 \times 3 \mathrm{~cm}^{2}\right)$ were made on a back of each Yorkshire pig symmetrically, consisting of 18 wounds on each side of the midline. The depth of each wound was to the depth of the muscle fascia to mimic scar formation. The interval of each wound was maintained between $3-5 \mathrm{~cm}$ to control the influence of the wounds on the process of wound healing. Both IV antibiotics and wound dressings with TegaDerm (3M, St Paul, MN, USA) were administered postoperatively for 5 days, and then an open dressing was maintained. Fifty days later, scar tissues distinct from peripheral normal tissues formed (Fig. 1) [12].


Fig. 1. (A) Pig scar model and scar formation. (A) A total of 36 fullthickness skin defects $\left(3 \times 3 \mathrm{~cm}^{2}\right)$ were made on the backs of Yorkshire pigs symmetrically, consisting of 18 wounds on each side of the midline. (B) Fifty days later, scar tissues distinct from peripheral normal tissues formed.

\section{Production of relaxin-expressing adenoviruses}

Relaxin-expressing and E1- and E3-deleted adenovirus vectors were produced according to the conventional method [8,10,12-14]. To develop an adenovirus encoding arginylglycylaspartic acid (RGD) consisting of the 9-amino acid sequence (CDCRGDCFC) between the HI-loop of the fiber knob, two complementary oligonucleotides encoding Arg-Gly-Asp were first synthesized and annealed to form a DNA duplex. This DNA duplex was designed to contain a BamHI overhang on the $5^{\prime}$ end and an MroI overhang on the $3^{\prime}$ end. The oligonucleotide sequences were 5 '-gatccTGTGACTGCCGCGGAGACTGTTTCTGCt-3' and 5'-ccggaACAATGACGGCGCCTCTGACAAAGACGg-3'. The annealed DNA duplex was then digested with $\mathrm{NcoI}$ and $\mathrm{MfeI}$ and cloned into pSK5543, generating a pSK (5543-RGD) adenovirus fiber shuttle vector.

The pSK (5543-RGD) shuttle vector was then linearized with SacI and XmnI, and the lacZ-expressing adenoviral vector pdE1/ lacZ was linearized with SpeI for homologous recombination in Escherichia coli BJ5183, resulting in the pdE1-RGD/lacZ adenoviral vector. To construct an adenovirus expressing lac $Z$ and RLX at the E1 and E3 regions, respectively, pdE1-RGD/lacZ was linearized with SpeI and co-transformed into Escherichia coli BJ5183 
with the PVuI-digested pSP72-E3/CMV-RLX shuttle vector for homologous recombination, producing the pdE1-RGD/lacZ/RLX adenoviral vector. All adenoviruses were developed and multiplied in 293 cells. Viral particle numbers were calculated from measurements of absorbance at $260 \mathrm{~nm}$, where labsorbency unit is equivalent to $10^{12}$ viral particles per milliliter $[8,10,12-14]$. Virus was diluted with phosphate buffered saline (PBS) to $5 \times 10^{7} \mathrm{PFU}[15]$.

\section{Injection of adenovirus}

On the 50th postoperative day, wound epithelialization and scar formation occurred. Anesthesia was induced in each pig by an intramuscular injection of Zoletil (5 mg/kg) and Rompun (2 mg/ $\mathrm{kg}$ ); all hairs were then removed from both the back and belly of each pig. Virus injection was performed using a 27-gauge needle and a 1-mL syringe, and adenoviruses were injected into the intradermal layer of the scar regions. Scars were divided into two groups; $5 \times 10^{7}$ PFU relaxin-expressing adenoviruses vectors (dE1RGD/LacZ/RLX) were injected into the experimental group (RLX). The same amount of adenoviruses not containing the relaxin gene (dE1-RGD/LacZ) was injected into the control group under the same conditions.

\section{Evaluation}

The surface area, color, and pliability of scars were compared and evaluated in the two groups. Evaluation was performed every 10 days until the 50th day after virus injection. The surface areas of scars were compared by taking scar images using a digital camera. By using $1 \mathrm{~cm}^{2}$ as the standard, measured with a ruler on the image, the surface areas of the scars were compared and assessed using Image J software (National Institutes of Health, USA). A spectrophotometer (CM-700D; KONICA MINOLTA, INC., Tokyo, Japan) was used to quantitatively analyze the color of the scars. The color of each scar was analyzed using melanin (the degree of scar darkness) and erythema (the degree of scar redness) indices [15]. Pliability was measured using a durometer (H1000 Mini-Dial, RexGauge Co., Buffalo Grove, IL, USA). Each value was measured more than three times, and their average value was used for analysis.

\section{Statistical analysis}

All test results were presented as the means \pm standard error of the mean (SEM), and the results of the quantitative analysis were statistically analyzed in the two groups using t-test. A p-value less than $0.05(p<0.05)$ was considered statistically significant. Statistical analysis was performed using SAS software (ver. 9.1.3, SAS Institute Inc., Cary, NC, USA).

\section{RESULTS}

\section{Scar area}

After virus injection, decreases were seen in the surface areas of scars in all groups over time. However, a more significant decrease in scar area was found in the RLX group compared with the control group (Fig. 2). The sizes of the initial scar were $3.15 \pm 0.15 \mathrm{~cm}^{2}$ and $3.12 \pm 0.11 \mathrm{~cm}^{2}$ in the RLX and control groups, respectively. However, the sizes were $1.70 \pm 0.10 \mathrm{~cm}^{2}$ in the control group 50 days after virus injection. The size was reduced to $1.37 \pm 0.05 \mathrm{~cm}^{2}$ in the RLX group, which was a significant decrease $(p<0.05)$ (Fig. 3).

\section{Scar color}

Immediately after scar formation, erythema indices representing the redness of the scar were $2.01 \pm 0.10$ and $2.01 \pm 0.09$ in the control and RLX groups, respectively, with no significant differences between the two groups. Fifty days after virus injection, erythema indices were $2.05 \pm 0.08$ and $1.52 \pm 0.15$, respectively, in the control and RLX groups (Fig. 4). Moreover, melanin indices indicating the degree of scar darkness of scar were in $0.23 \pm 0.02$ and $0.23 \pm 0.03$ in the control and RLX groups, respectively, with no significant differences between the two groups. Fifty days after virus injection, melanin indices were $0.21 \pm 0.04$ and $0.08 \pm 0.02$, respectively, in the control and RLX groups (Fig. 5). No significant differences were shown between the erythema and melanin indices measured immediately after scar formation and 50 days after injection using spectrophotometry in the control groups. In contrast, significant decreases were seen in the erythema and melanin indices in the RLX group $(p<0.05)$. 

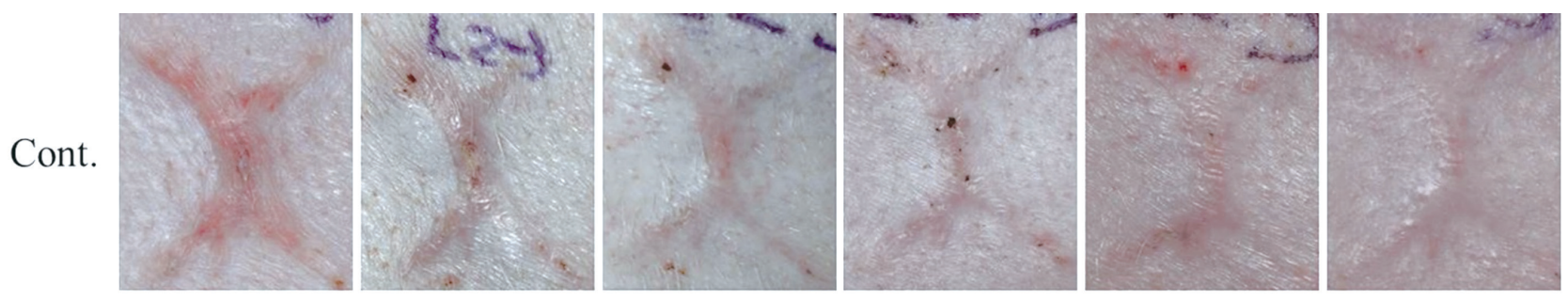

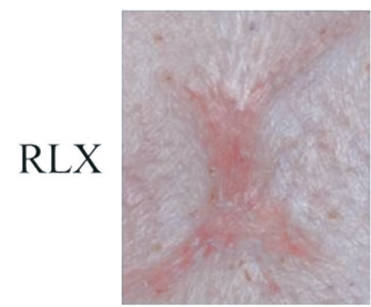

Immediately after virus injection

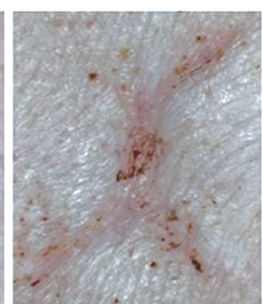

10 days after virus injection

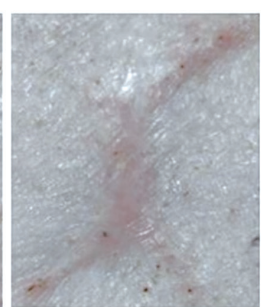

20 days after virus injection

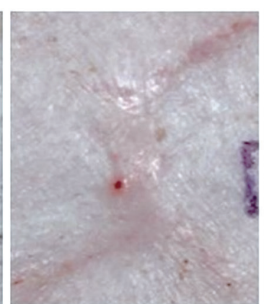

30 days after virus injection

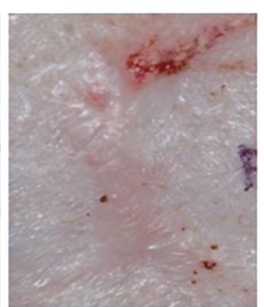

40 days after virus injection

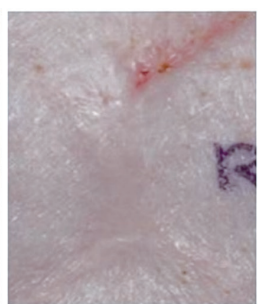

50 days after virus injection

Fig. 2. Scar surface change. The surface area of the scars decreased in all groups and persisted for 50 days after virus injection. A greater decrease was observed in the RLX group. Cont., control group; RLX, relaxin group.

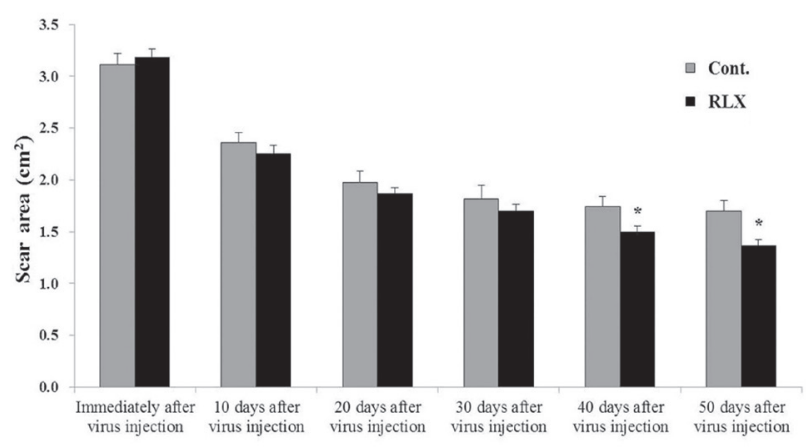

Fig. 3. Scar surface area. The sizes of the initial scar were $3.15 \pm 0.15 \mathrm{~cm}^{2}$ and $3.12 \pm 0.11 \mathrm{~cm}^{2}$ in the RLX and control groups, respectively. The scar sizes were $1.70 \pm 0.10 \mathrm{~cm}^{2}$ in the control group 50 days after virus injection. The size was reduced to $1.37 \pm 0.05 \mathrm{~cm}^{2}$ in the RLX group, which was a significant decrease $\left({ }^{*} p<0.05\right)$. RLX, relaxin group; Cont., control group.

\section{Scar pliability}

The pliability of the scars increased as evaluated durometry values decreased. Immediately after the formation of the scar, durometry values were $19.73 \pm 0.88$ and $19.08 \pm 1.06$ in the control and RLX groups, respectively, with no significant differences. Fifty days after injection of virus, the values decreased to $17.46 \pm 1.62$ and 11.15 \pm 1.72 , respectively, in the control and RLX groups. Pliability increased in all groups over time. Pliability significantly increased

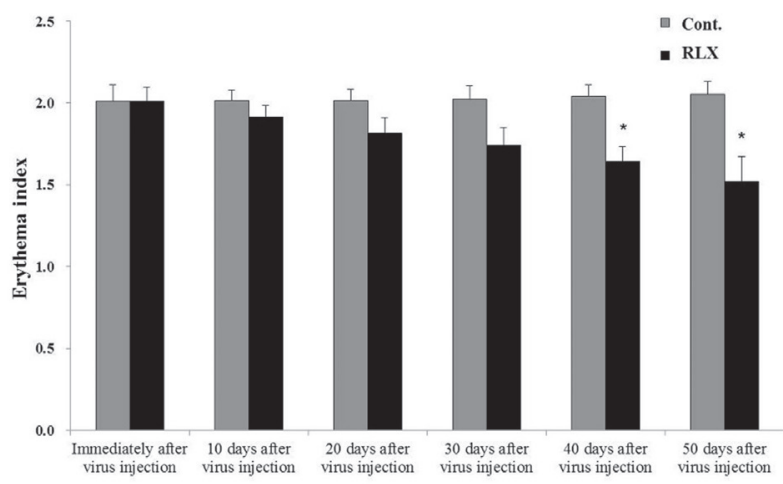

Fig. 4. Erythema index. Immediately after scar formation, erythema indices were $2.01 \pm 0.10$ and $2.01 \pm 0.09$ in the control and RLX groups, respectively. Fifty days after virus injection, erythema indices were $2.05 \pm 0.08$ and $1.52 \pm 0.15$, respectively, in the control and RLX groups $\left({ }^{*} p<0.05\right)$. RLX, relaxin group; Cont., control group.

in the RLX group compared with control groups $(p<0.05)$ (Fig. 6).

\section{DISCUSSION}

Scars developing after a trauma or surgery incur a large number of functional problems. With improvements in one's economic status, many patients want to minimize scar formation and enhance wound healing. For this reason, a large number of preservative treatments, including scar revision and laser therapy, have 


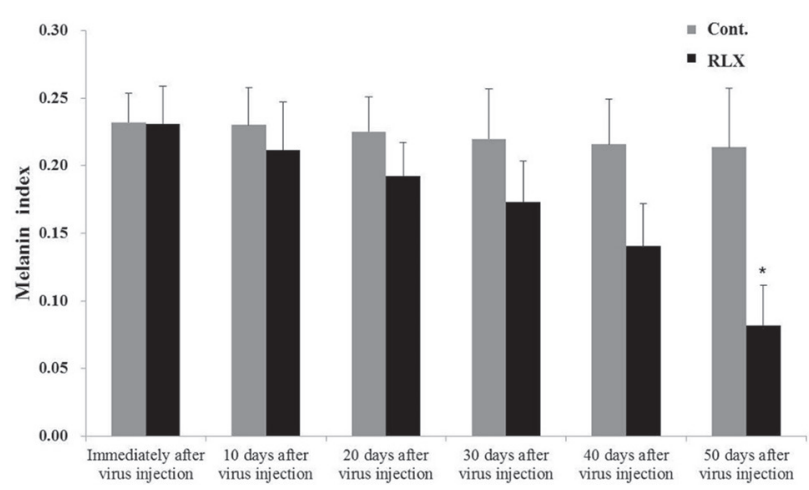

Fig. 5. Melanin index. Melanin indices were in $0.23 \pm 0.02$ and $0.23 \pm 0.03$ in the control and RLX groups, respectively, with no significant differences between the two groups. Fifty days after virus injection, melanin indices were $0.21 \pm 0.04$ and $0.08 \pm 0.02$, respectively, in the control and RLX groups $\left({ }^{*} p<0.05\right)$. RLX, relaxin group; Cont., control group.

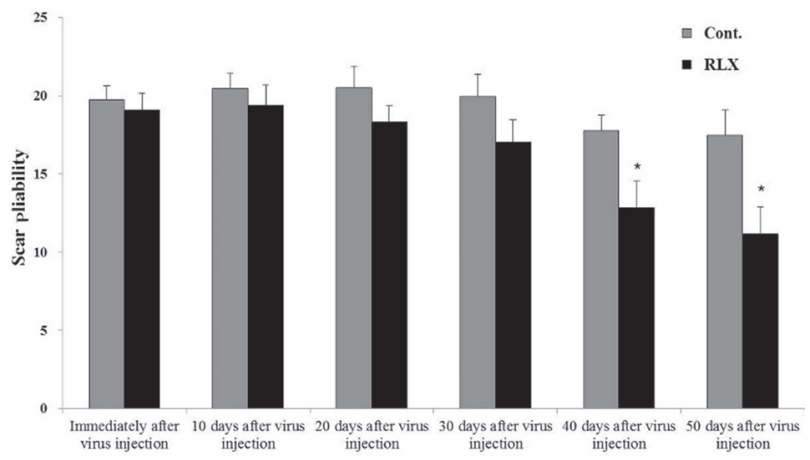

Fig. 6. Scar Pliability. The pliability of the scars increased as evaluated durometry values decreased. Immediately after the formation of the scar, durometry values were $19.73 \pm 0.88$ and $19.08 \pm 1.06$ in the control and RLX groups, respectively, with no significant differences. Fifty days after injection of virus, the values decreased to $17.46 \pm 1.62$ and $11.15 \pm 1.72$, respectively, in the control and RLX groups $\left({ }^{*} p<0.05\right)$. RLX, relaxin group; Cont., control group.

been applied to prevent scar formation. However, the successful treatment of scars has not been fully realized [16-18].

Several factors are involved in the formation of a scar. Continued inflammation in a wound increases the expression of TGF- $\beta 1$, which leads to the accelerated differentiation of fibroblasts into myofibroblasts. The major cause of fibrotic scar contraction is considered the elevated $\alpha$-SMA levels in myofibroblasts. Furthermore, TGF- $\beta 1$ is believed to be involved in abnormal fibrosis by synthesizing extracellular matrix and infiltrating the interstitial tissues [18-23].

Mast cells, also involved in the process of wound healing, are reported to be involved in the proliferation and contraction of fibroblasts, and the synthesis of extracellular matrix. They are also thought to play a crucial role in scar formation. The amount of mast cells was four times higher in hypertrophic scars than in normal skin tissues [24-26].

Animal models are essential for advances in scar improvement. However, scars are rarely formed in animals, unlike in humans. We have created an animal scar model by making full-thickness skin defects on the backs of pigs that are similar to human scars [27-30]. In this study, we used human relaxin-expressing adenoviruses in the pig scar model. Both human and porcine relaxin showed similar biological activity in a previous study [31]. Additionally, human relaxin was also effective in a porcine scar model [32].

We carried out a relaxin gene therapy study on keloids and local ischemic flaps using relaxin-expressing adenoviruses [12-14]. Relaxin gene therapy on keloids was the previous stage of this study. Relaxin is anticipated to have positive effects on the treatment and prevention of keloids by reducing the expression of collagen I and III, both excessively expressed in keloids, and by decreasing the levels of elastin and fibronectin, which are both increased in keloids.

This study was performed using an in vivo experimental model by adopting a pig scar model. The aim of this study was to verify the anti-fibrotic effect of relaxin on scars. When relaxin-expressing adenoviruses were injected into pig scars, the reduction of the surface area of the scar was accelerated clinically and skin color was normalized. Moreover, the flexibility of the scar was improved.

These improvements of scars were believed to be caused by inhibiting TGF- $\beta 1$ expression and decreasing the number of mast cells, which play crucial roles in scar formation. The inhibition of TGF- $\beta 1$ by relaxin in scar tissues was reported previously $[8,12,13,19]$. Relaxin was shown to decrease mast cell numbers in a swine model of acute myocardial infarction $[33,34]$. The inhibition of TGF- $\beta 1$ and the decrease in mast cell number by relaxin may prevent the conversion of fibroblasts into myofibroblasts during the scar remodeling process.

This study has several limitations as a preliminary study. First, 
inherent to an animal study is the question of whether the same effect would be observed in human. Second, we obtained the results for 50 days after viral injection. The duration of 50 days would be short to evaluate the entire process of scar maturation and remodeling. Third, all results in this study were obtained through the gross findings as surface area, color and pliability of scars. Further studies including histological study are required to find the definite correlation with relaxin and scar remodeling. Additionally, the investigation for the mechanism of relaxin in scar remodeling would be useful.

\section{REFERENCES}

1. Lorenz HP. Wound healing: Repair biology and wound and scar treatment. In: Mathes SJ, editor. Mathes Plastic surgery. 2nd ed. Philadelphia: Saunders; 2006. p. 209-35.

2. Desmouliere A, Darby IA, Gabbiani G. Normal and pathologic soft tissue remodeling: role of the myofibroblast, with special emphasis on liver and kidney fibrosis. Lab Invest 2003;83:1689-707.

3. Samuel CS, Hewitson TD, Unemori EN, Tang ML. Drugs of the future: the hormone relaxin. Cell Mol Life Sci 2007;64:1539-57.

4. Sherwood OD. Relaxin's physiological roles and other diverse actions. Endocr Rev 2004;25:205-34.

5. Samuel CS, Hewitson TD. Relaxin in cardiovascular and renal disease. Kidney Int 2006;69:1498-502.

6. Dschietzig T, Bartsch C, Baumann G, Stangl K. Relaxin-a pleiotropic hormone and its emerging role for experimental and clinical therapeutics. Pharmacol Ther 2006;112:38-56.

7. Unemori EN, Erikson ME, Rocco SE, Sutherland KM, Parsell DA, Mak J, et al. Relaxin stimulates expression of vascular endothelial growth factor in normal human endometrial cells in vitro and is associated with menometrorrhagia in women. Hum Reprod 1999;14:8006.

8. Kim JH, Lee YS, Kim H, Huang JH, Yoon AR, Yun CO. Relaxin expression from tumor-targeting adenoviruses and its intratumoral spread, apoptosis induction, and efficacy. J Natl Cancer Inst 2006;98:1482-93.

9. Unemori EN, Lewis M, Constant J, Arnold G, Grove BH, Normand J, et al. Relaxin induces vascular endothelial growth factor expression and angiogenesis selectively at wound sites. Wound Repair Regen 2000;8:361-70.

10. Kim J, Cho JY, Kim JH, Jung KC, Yun CO. Evaluation of E1B gene-attenuated replicating adenoviruses for cancer gene therapy. Cancer Gene Ther 2002;9:725-36.

11. Chartier C, Degryse E, Gantzer M, Dieterle A, Pavirani A, Mehtali M. Efficient generation of recombinant adenovirus vectors by homologous recombination in Escherichia coli. J Virol 1996;70:4805-10.
12. Lee WJ, Kim YO, Choi IK, Rah DK, Yun CO. Adenovirus-relaxin gene therapy for keloids: implication for reversing pathological fibrosis. Br J Dermatol 2011;165:673-7.

13. Lee WJ, Choi IK, Lee JH, Lee JS, Kim YO, Rah DK, et al. Relaxin-expressing adenovirus decreases collagen synthesis and up-regulates matrix metalloproteinase expression in keloid fibroblasts: in vitro experiments. Plast Reconstr Surg 2012;130:407e-417e.

14. Lee WJ, Yun CO, Yun IS, Kim YO, Choi IK, Yun TJ, et al. Augmentation of rat skin flap viability by relaxin-expressing adenovirus. Wound Repair Regen 2011;19:709-17.

15. Yun IS, Lee WJ, Rah DK, Kim YO, Park BY. Skin color analysis using a spectrophotometer in Asians. Skin Res Technol 2010;16:311-5.

16. Park BY, Shin IS, Yun IS. Dovetail scar revision. Dermatol Surg 2012;38:1716-21

17. Kim SG, Kim EY, Kim YJ, Lee SI. The efficacy and safety of ablative fractional resurfacing using a 2,940-Nm Er:YAG laser for traumatic scars in the early posttraumatic period. Arch Plast Surg 2012;39:232-7.

18. Greenhalgh DG. Consequences of excessive scar formation: dealing with the problem and aiming for the future. Wound Repair Regen 2007;15 Suppl 1:S2-5.

19. Shah M, Foreman DM, Ferguson MW. Neutralising antibody to TGF-beta 1,2 reduces cutaneous scarring in adult rodents. J Cell Sci 1994;107(Pt5):1137-57.

20. Wilgus TA, Vodovotz Y, Vittadini E, Clubbs EA, Oberyszyn TM. Reduction of scar formation in full-thickness wounds with topical celecoxib treatment. Wound Repair Regen 2003;11:25-34.

21. Liu W, Chua C, Wu X, Wang D, Ying D, Cui L, et al. Inhibiting scar formation in rat wounds by adenovirus-mediated overexpression of truncated TGF-beta receptor II. Plast Reconstr Surg 2005;115:860-70.

22. Gallant CL, Olson ME, Hart DA. Molecular, histologic, and gross phenotype of skin wound healing in red Duroc pigs reveals an abnormal healing phenotype of hypercontracted, hyperpigmented scarring. Wound Repair Regen 2004;12:305-19.

23. Margulis A, Nocka KH, Wood NL, Wolf SF, Goldman SJ, Kasaian MT. MMP dependence of fibroblast contraction and collagen production induced by human mast cell activation in a three-dimensional collagen lattice. Am J Physiol Lung Cell Mol Physiol 2009;296:L23647.

24. Kitamura Y, Oboki K, Ito A. Molecular mechanisms of mast cell development. Immunol Allergy Clin North Am 2006;26:387-405.

25. Sur R, Cavender D, Malaviya R. Different approaches to study mast cell functions. Int Immunopharmacol 2007;7:555-67.

26. Gilfillan AM, Rivera J. The tyrosine kinase network regulating mast cell activation. Immunol Rev 2009;228:149-69.

27. Yun IS, Jeon YR, Lee WJ, Lee JW, Rah DK, Tark KC, et al. Effect of human adipose derived stem cells on scar formation and remodeling in a pig model: a pilot study. Dermatol Surg 2012;38:1678-88.

28. Corr DT, Gallant-Behm CL, Shrive NG, Hart DA. Biomechanical behavior of scar tissue and uninjured skin in a porcine model. Wound Repair Regen 2009;17:250-9.

29. Wang XQ, Kravchuk O, Liu PY, Kempf M, Boogaard CV, Lau P, et al. 
The evaluation of a clinical scar scale for porcine burn scars. Burns 2009;35:538-46.

30. Wang XQ, Liu PY, Kempf M, Cuttle L, Chang AH, Wong M, et al. Burn healing is dependent on burn site: a quantitative analysis from a porcine burn model. Burns 2009;35:264-9.

31. Zhao S, Lee HY, Sherwood OD. Porcine and human relaxin bioactivity: bioactivities of porcine relaxin and human relaxin do not differ in mice and rats. Ann N Y Acad Sci 2005;1041:126-31.

32. Perna AM, Masini E, Nistri S, Bani Sacchi T, Bigazzi M, Bani D. Human recombinant relaxin reduces heart injury and improves ventric- ular performance in a swine model of acute myocardial infarction. Ann N Y Acad Sci 2005;1041:431-3.

33. Nistri S, Cinci L, Perna AM, Masini E, Mastroianni R, Bani D. Relaxin induces mast cell inhibition and reduces ventricular arrhythmias in a swine model of acute myocardial infarction. Pharmacol Res 2008;57:43-8.

34. Nistri S, Cinci L, Perna AM, Masini E, Bani D. Mast cell inhibition and reduced ventricular arrhythmias in a swine model of acute myocardial infarction upon therapeutic administration of relaxin. Inflamm Res 2008;57 Suppl 1:S7-8 